\begin{abstract}
Title of Thesis:

PREDICTORS AND MODERATORS OF PARENT ENGAGEMENT IN EARLY INTERVENTIONS FOR BEHAVIORALLY INHIBITED PRESCHOOL-AGED CHILDREN

Danielle Ruth Novick, Master of Science, 2019

Thesis Directed By:

Dr. Andrea Chronis-Tuscano, Professor, Department of Psychology
\end{abstract}

Behavioral inhibition (BI) is a temperamental style that poses risk for later anxiety. Efficacious interventions have been developed for inhibited children, but their success depends on parent engagement. However, little is known regarding predictors of parent engagement in early interventions for BI. This study examined parent-, child- and treatment-level (i.e., parent-only or parent-child) factors as independent and interactive predictors of parent engagement (attendance, and parentreported homework completion and treatment satisfaction) in a randomizedcontrolled trial comparing two interventions for inhibited preschoolers $(N=151)$. Results suggest that child anxiety may motivate parent engagement, particularly when children receive concurrent treatment and/or in-vivo coaching. However, intensive treatment may be too burdensome for depressed parents, whereas less intensive treatments may be more acceptable to non-anxious parents of anxious children. 


\title{
PREDICTORS AND MODERATORS OF PARENT ENGAGEMENT IN EARLY INTERVENTIONS FOR BEHAVIORALLY INHIBITED PRESCHOOL-AGED CHILDREN
}

\author{
by \\ Danielle Ruth Novick \\ Thesis submitted to the Faculty of the Graduate School of the \\ University of Maryland, College Park, in partial fulfillment \\ of the requirements for the degree of \\ Master of Science \\ 2019
}

Advisory Committee:

Professor Andrea Chronis-Tuscano, Chair

Professor Lea Dougherty

Professor Kenneth Rubin 
(C) Copyright by

Danielle Ruth Novick 2019 


\section{Table of Contents}

Table of Contents ..........................................................................................ii

Chapter 1: Introduction .................................................................................. 1

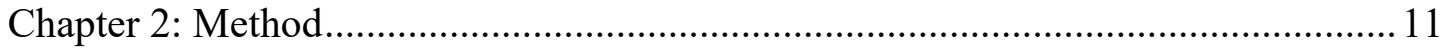





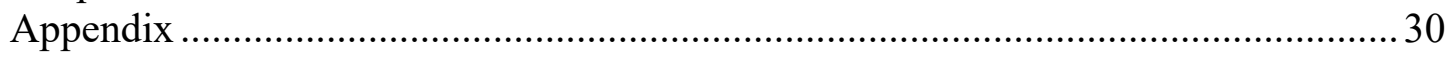






\section{Chapter 1: Introduction}

Characterized by expressions of fear, social reticence, and increased arousal in the face of novel stimuli (Kagan, Reznick, Snidman, Gibbons, \& Johnson, 1988), behavioral inhibition (BI) is a heritable temperamental style that is observed in 15$20 \%$ of infants (Degnan \& Fox, 2007). Youth who exhibit stability in BI over time are at increased risk for the development of anxiety, specifically social anxiety disorder (Chronis-Tuscano et al., 2009). Nevertheless, only approximately half of those identified as behaviorally inhibited in childhood ultimately develop anxiety disorders (Degnan \& Fox, 2007), suggesting that external and internal factors may influence certain inhibited children's vulnerability for the development of anxiety.

\section{Theoretical Model}

In their theoretical model, Rubin, Coplan, and Bowker (2009) suggest that interactions with parents and peers serve to mitigate or exacerbate negative outcomes over time for youth with elevated BI. During infancy and toddlerhood, parents begin to perceive their inhibited children as vulnerable in the face of novelty, and consequently respond to their children in overprotective ways (e.g., less autonomy granting; reinforcing anxious behaviors; Rubin et al., 2009). Further, inhibited children elicit such anxiolytic behaviors from their parents, forming a transactional cycle over time. This cycle has been shown to put inhibited children at greater risk of experiencing peer victimization, social exclusion, and rejection (Rubin et al., 2009). Inhibited children's social failures and subsequent negative self-perceptions serve to further exacerbate $\mathrm{BI}$ and heighten risk for internalizing disorders such as anxiety and depression (Rubin et al., 2009). 


\section{Parent Engagement in Child Treatment}

The malleability of these risk factors and the high degree to which young children depend on their parents for their emotional and physical needs make parenting a natural target of early intervention programs for children high in BI (Chronis-Tuscano, Danko, Rubin, Coplan, \& Novick, 2018). Much of the treatment literature has focused on cognitive behavioral therapy (CBT) for older youth with anxiety. However, since CBT involves the use of higher-order cognitive skills (i.e., executive functioning skills, metacognition, language abilities) that most preschoolage children have not yet developed (Carpenter, Puliafico, Kurtz, Pincus, \& Comer, 2014), early interventions tend to be more behavioral in nature and require greater parent involvement. Indeed, the majority of interventions for young children with $\mathrm{BI} /$ anxiety have taken the form of parent training (PT; Cartwright-Hatton et al., 2011), an evidence-based approach originally developed for childhood externalizing problems that focuses on the parent's role in the development and continuity of child behavior. PT interventions equip parents with behavior modification techniques that are grounded in social learning theory (Serketich \& Dumas, 1996). Therapists teach parents to effectively respond to problematic child behaviors and to increase desirable behaviors using differential positive reinforcement. In PT for children with $\mathrm{BI} /$ anxiety, parents learn to reinforce brave, approach behaviors (as opposed to avoidance), refrain from reinforcing anxious or avoidant behaviors, and implement exposures to anxiety-provoking stimuli between sessions (Chronis-Tuscano et al., 2018). 
Despite promising evidence that behavioral and/or cognitive-behavioral prevention/intervention programs for children with or at-risk for anxiety are efficacious, the success of the majority of such programs depends on parent engagement. Parents with poor participation in PT lack opportunities to learn and practice important skills hypothesized to reduce risk for child anxiety, thus impeding positive treatment outcomes. Indeed, research convincingly demonstrates the link between parent engagement in child treatment and treatment outcomes across interventions for an array of childhood psychosocial difficulties (Haine-Schlagel \& Walsh, 2015). However, there is some lack of clarity regarding the definition of "engagement" across the literature.

Engagement in treatment encompasses both a behavioral and an attitudinal component. The behavioral component refers to actions necessary to pursue, attend, and participate in treatment (Staudt, 2007). Although session attendance is often examined as a sole index of the behavioral component, attendance alone may not be sufficient in demonstrating the degree to which parents are fully engaged in child treatment. Results of numerous studies indicate that other measures of parent engagement, such as between-session homework completion, are stronger predictors of outcomes than session attendance alone (Nix, Bierman, \& McMahon, 2009). The attitudinal component refers to the intent to invest in treatment and the belief that treatment will be or was beneficial or relevant. Treatment acceptability has been used as an index of this component and captures individuals' beliefs regarding the degree to which treatment is satisfactory, relevant, and meets expectations (Calvert \& Johnston, 1990). Parent and child ratings of treatment acceptability have been shown 
to be positively associated with treatment adherence (Miller \& Kelley, 1992) and outcomes (Kazdin, 2000).

\section{Predictors of Engagement in Parent Training for Child Externalizing Problems}

Despite the important link between parent engagement in PT and optimal treatment outcomes, the majority of parent engagement research has focused on PT for externalizing problems. This research largely suggests that low SES, ethnic minority status, and single parenting predict less treatment engagement in PT for child externalizing problems (Haine-Schlagel \& Walsh, 2015). Further, while families with more severe child symptomatology have been shown in some studies to be less likely to engage in PT (Haine-Schlagel \& Walsh, 2015), others have found child symptom severity to predict greater engagement, perhaps due to a greater perceived need for treatment (Reid, Webster-Stratton, \& Baydar, 2004). Interestingly, Nix and colleagues (2009) found that child symptom severity predicted parent engagement in PT as measured by homework completion, but not attendance, highlighting the value of examining multiple indices of engagement. Similarly, parental psychopathology (most often defined as maternal depression) has been linked to poorer engagement in PT for child externalizing problems (Haine-Schlagel \& Walsh, 2015), but this relation has not consistently been found across studies (Prinz \& Miller, 1994).

\section{Predictors of Parent Engagement in Child Anxiety Treatments}

Although predictors of parent engagement in treatments for child externalizing problems are well-documented, similar research on treatments targeting child internalizing symptoms (e.g., anxiety) remains sparse. Further, despite the persistent course of early-onset anxiety disorders and support for existing treatments 
for young children with or at-risk for anxiety (Chronis-Tuscano et al., 2018), the majority of the extant literature investigating engagement in treatments targeting child anxiety tends to focus on CBT for school-age youth. Though some studies suggest that families from ethnic minority backgrounds as well as those with more severe child baseline symptoms are more likely to drop out of CBT for child anxiety (e.g., Kendall \& Sugarman, 1997), others do not demonstrate the same relation (e.g., Waters, Ford, Wharton, \& Cobham, 2009). Additionally, examination of parent factors predicting parent engagement in CBT for child anxiety is limited, and even more so regarding treatments for young children at risk for anxiety by virtue of BI. Though research has suggested a link between higher parental stress, anxiety, and depression, and lower treatment satisfaction with treatment attrition (Waters et al., 2009), other studies have not demonstrated an association between parent psychopathology and attrition from CBT for child anxiety (Kendall \& Sugarman, 1997). There are also variable findings regarding the relations between SES, treatment satisfaction, single-parent status and parent engagement in child anxiety treatment (Kendall \& Sugarman, 1997; Podell \& Kendall, 2011; Pereira et al., 2016). Much like the research examining PT for child externalizing, these studies demonstrate inconsistent findings regarding the role of child and parent factors in predicting engagement in child anxiety treatment.

Furthermore, the role of both parents' engagement in treatment has been neglected across the extant research examining interventions for child internalizing and externalizing. Research suggests that children who have two parents participate in PT for externalizing problems demonstrate significantly better treatment outcomes 
than youth who only have one parent participate (Bagner \& Eyberg, 2003). Though it is possible that children with internalizing problems may also experience greater treatment gains when both parents can support each other in implementing exposures outside of session, research on co-parent engagement remains sparse. Indeed, maternal engagement in child treatment is often misrepresented as parent engagement, highlighting a paucity of father involvement in child treatment research. Failing to examine father involvement in child treatments neglects findings indicating that both mothers and fathers influence the development of child psychosocial difficulties (Connell \& Goodman, 2002). Further, evidence-based strategies to enhance parent involvement in child treatment will be limited if they solely account for patterns of maternal engagement. In one of the only studies to examine the link between mother and father engagement and child anxiety treatment outcomes, greater father attendance and combined mother and father attendance were associated with child treatment gains (Podell \& Kendall, 2011). In light of the dearth of extant studies examining the engagement of both parents in child anxiety treatment, such findings underscore the critical need to further understand how to best engage co-parents.

\section{Treatment Factors Associated with Parent Engagement in Child Treatment}

Across the treatment literature, few researchers have examined treatment factors that predict parent engagement in treatments for young children. Given that many parents may believe that their children should directly participate in treatments (Tynan, Schuman, \& Lampert, 1999), researchers have aimed to enhance parent engagement in PT through the inclusion of a concurrent child treatment component. Several studies demonstrate that PT targeting externalizing problems with a 
concurrent child group enhances homework completion, attendance, and treatment satisfaction (e.g., Fabiano et al., 2009), other studies have yielded null results (e.g., Loren, Ciesielski, \& Tamm, 2017).

Although existing PT programs targeting child BI share many similarities, their differing formats may yield critical differences in treatment engagement. Nevertheless, researchers to date have yet to examine treatment format as a predictor of parent engagement in PT programs for young children at-risk for later anxiety. Numerous studies have, however, indicated that parents tend to exhibit higher rates of engagement in CBT for anxiety in older children when enrolled in programs involving both parents and children as opposed to parent-only formats.

Multiple comparisons of parent-only and parent-child group CBT demonstrate that families in the parent-only group prematurely terminate treatment marginally more than those in the parent-child group (Monga, Rosenbloom, Tanha, Owens, \& Young, 2015; Waters et al., 2009). Moreover, Waters and colleagues (2009) did not find any significant differences in child anxiety disorder status between parent-child and parent-only CBT groups at post-treatment nor at one year follow up, suggesting the treatments themselves worked comparably. Although child involvement in treatment was not necessary to achieve positive treatment gains, families appeared to remain in treatment more when both parents and children participated. Given the potential value of concurrent child groups, examining the link between treatment format and parent engagement in treatments targeting child $\mathrm{BI}$ and anxiety may be quite informative. Directly addressing impairment in the peer context through the inclusion of a concurrent child group may be especially relevant to the presenting 
problems of inhibited or socially anxious children, and may thus have critical implications for parent engagement.

\section{Gaps in the Literature}

Although there has been substantial headway in the development of early interventions for young children with BI/anxiety, researchers have yet to examine factors associated with engagement in PT for young children with $\mathrm{BI} /$ anxiety. Predictors of parent engagement in PT are likely to differ from parent engagement in CBT for older youth given the need for increased and direct parent involvement in PT for younger children. Moreover, little is known regarding the link between parental anxiety and parent engagement in child treatment. Parents of anxious children are more likely to experience symptoms of anxiety themselves (Last, Hersen, Kazdin, Orvaschel, \& Perrin, 1991), which may impede (or enhance) parent engagement. Furthermore, the majority of studies examining predictors of parent engagement in treatment for child anxiety have been limited to investigations of parental involvement within treatment sessions (i.e., therapist-rated engagement during the session, attendance/attrition) with little research on engagement outside of therapy sessions (i.e., homework completion).

Between-session homework is widely considered a fundamental component of CBT for adults (Kazantzis, Whittington, \& Dattilio, 2010). Despite variable findings regarding the value of homework completion in CBT for anxious youth (Arendt, Thatsum, \& Hougaard, 2016; Pereira et al., 2016), researchers who have examined the effectiveness of adult CBT (Kazantzis et al., 2010) and PT for child externalizing (Nix et al., 2009) suggest that homework completion is critical to positive treatment 
gains. Likewise, there is little research regarding predictors of treatment satisfaction in early interventions targeting child anxiety. Additionally, few studies capture predictors of co-parent engagement, with the majority of the extant studies focusing on the primary parent's engagement or utilizing a global measure of both parents' engagement. Lastly, few studies to date have gone beyond parent and child factors to identify treatment factors associated with parent engagement, or to examine how these factors interact with one another. Indeed, researchers have cautioned against the assumption that predictors of engagement are uniform across treatment modalities (i.e., parent-only vs. parent-child; Armbruster \& Kazdin, 1994), and treatment format may moderate the degree to which certain factors predict parent engagement. Identifying factors that predict parental engagement differentially across intervention formats for children high in BI may have the potential to guide efforts to enhance parental involvement.

\section{Study Aims and Hypotheses}

The current study addresses these gaps by examining the interaction between child, parent, and treatment factors as predictors of three components of parent engagement (attendance, homework completion, and treatment satisfaction) in a randomized controlled trial (RCT) examining two early intervention programs for inhibited preschool-age children: The Turtle Program, an 8-session intervention comprised of concurrent parent and child groups (Chronis-Tuscano et al., 2015; Danko, O’Brien, Rubin, \& Chronis-Tuscano, 2019), and Cool Little Kids, a 6-session, parent-only group intervention (Rapee, Kennedy, Ingram, Edwards, \& Sweeney, 2005). We hypothesized that parents in the Turtle Program would exhibit and report 
greater treatment engagement relative to parents receiving CLK. We also expected that greater child anxiety and less parent psychopathology would each independently predict greater parent engagement. Further, we hypothesized that higher parent lifetime anxiety would interact with higher child anxiety children to predict more treatment engagement in the Turtle Program. Given the research indicating that parent depression predicts less engagement in child treatment, we also examined the unique contributions of lifetime parent depression severity as a predictor of engagement. Finally, given the dearth of research regarding predictors of co-parent treatment engagement, we also explored the interaction between child anxiety, primary parent anxiety, and co-parent anxiety in predicting co-parent engagement. 


\section{Chapter 2: Method}

\section{Participants}

Participants included 151 children aged 45-64 months and their custodial caregivers. Child, primary parent (PP), and co-parent (CP) demographic information is presented in Table 1. Within families in which only one parent participated in the study, that parent was specified as the PP. Within families in which two parents participated in the study, the parent who reported spending the most time engaged in caregiving for the child and/ or committed to attending all treatment sessions was specified as the PP; the other was specified as the CP. Of the 127 CPs who consented to participate in the study, only the $116(\sim 91 \%)$ CPs who described themselves as currently married (as opposed to divorced, separated, or never married) were included in analyses examining CP engagement. Unmarried, separated, or divorced families were excluded from these CP engagement analyses, as we have a limited understanding of the degree to which CPs are involved in their children's lives within these families and their co-parenting relationship may meaningfully differ from that of married parents. Five CPs did not provide demographic information.

Eligible children were required to be between 45-64 months of age, attend a structured school setting (e.g., daycare, preschool), and to have at least one custodial parent who consented to participate. Parents and their children were only eligible to participate in a pre-treatment lab visit if their children scored in the $85^{\text {th }}$ percentile or above on the Behavioral Inhibition Questionnaire (BIQ; Bishop, Spence, \& MacDonald, 2003) and $\leq 15$ on the Social Communication Questionnaire to screen out autism spectrum disorders (SCQ; Eaves, Wingert, Ho, \& Mickelson, 2006) during 
the initial telephone screen. We administered both measures as part of our initial phone screen protocol. Children were excluded if they met criteria for selective mutism at baseline.

\section{Procedures}

Parents interested in participating in this RCT completed an approximately 30 minute telephone screen, during which we collected demographic information and assessed inclusion/exclusion criteria. Families who met initial inclusion criteria were invited to participate in a pre-treatment assessment. During the pre-treatment visit, research staff obtained informed consent, and parents completed lab tasks as well as a semi-structured diagnostic interview about their child. Parents also later completed a semi-structured diagnostic interview over the telephone to assess their own anxiety and depression. All interviews were audio-recorded and administered by independent evaluators who were unaware of treatment assignments. Eligible participants were block randomized to one of two treatment groups based on sex and the presence of a baseline anxiety diagnosis. Groups were matched on total therapy hours. Starting in the fifth cohort (of twelve cohorts), a subset of PPs $(n=101)$ completed a record of homework completion and the satisfaction survey at the post-treatment lab visit. There were no significant differences in parent or child age, gender, race, ethnicity, family income, and parent education between families who did and did not complete these engagement measures.

The Turtle Program. The Turtle Program ("Turtle") is an 8-session adaptation of parent-child interaction therapy (PCIT; Eyberg, 1988) and Social Skills Facilitated Play (SSFP; Coplan, Schneider, Matheson, \& Graham, 2010) for 
preschool-age children with elevated BI and their parents (described in ChronisTuscano et al., 2015; Danko et al., 2019). In the present study, 6-7 parents and their children typically participated in each of the 90-minute concurrent parent and child group sessions.

The parent group session material was divided into three phases. First, parents were taught Child Directed Interaction (CDI) skills, such as allowing the child to take the lead during play, differential attention, and positive reinforcement. Parent-child dyads were pulled from the parent and child groups so that parents could receive live, in-vivo coaching during one session of the CDI phase. These skills were practiced during a 5-minute play time ("special time") as daily homework. Meanwhile, the parent group observed the parent-child dyad via a television for the purpose of vicarious learning. The second phase of the program taught parents Bravery Directed Interaction (BDI) skills. These skills included implementing fear hierarchies, antecedent control, and positive reinforcement for social behaviors. Parents received live, in-vivo coaching during two sessions of the BDI phase in order to practice implementing exposures, often within the peer group context (e.g., show-and-tell, graduation party). The final phase of the program taught parents Parent Directed Interaction (PDI) skills, including behavior management techniques (e.g., effective commands and time out). Treatment integrity checks for the Turtle parent group demonstrated $98.99 \%$ adherence.

The child group, an elaboration of SSFP, engaged children in learning social problem-solving and emotion regulation skills by simulating situations in which children at this developmental stage struggle socially. Children learned skills in a 
developmentally appropriate format (e.g., puppets, games, stories) during a brief didactic circle time lesson lasting about 10 minutes. For the remainder of the session, children participated in free play as well as other activities that allowed for exposure to feared social situations and reinforcement for approach (vs. avoidant) behaviors. Treatment integrity checks for SSFP groups demonstrated $89.79 \%$ adherence.

Cool Little Kids. Cool Little Kids (CLK; Rapee et al., 2005), the active control group, is a 6-session, group-based psychoeducation program for parents of 3to 5-year-old children with elevated BI. Each parent-only treatment session lasted 120 minutes, and 6-7 families participated in each group. The initial two CLK sessions focused on teaching parents about the etiology of anxiety, recognizing signs of child anxiety, and constructive and unhelpful responses to child anxiety. The subsequent CLK sessions focused on teaching parents principles of exposure therapy as well as brief cognitive restructuring for parent anxiety. Treatment integrity checks demonstrated $97.57 \%$ adherence.

Turtle and CLK parent groups were both implemented by two therapists: a licensed clinical psychologist and/or clinical psychology doctoral students. The child group, SSFP, was implemented by two group leaders consisting of a human development doctoral student, post-baccalaureate research coordinator, and/or advanced undergraduate student. All treatment sessions were video-recorded, and licensed clinical psychologists provided supervision. Parent group leaders received training in PCIT from a Level II Trainer, in which parent and child group leaders met mastery criteria in CDI skills and observed all sessions of a previous cohort. 
Measures

Child Anxiety. The Anxiety Disorders Interview Schedule for Children for DSM-5-Parent Version (ADIS-P) was used to measure baseline levels of child anxiety. The ADIS-P assesses for clinical levels of impairment using a cliniciangenerated Clinician Severity Rating (CSR), ranging from 0-8. A 4 on this scale indicates clinically-significant impairment and need for treatment. Evaluators were trained on the ADIS-P through didactics, by conducting practice interviews, completing interviews with live supervision, and achieving reliability with clinical supervisors. For this study, baseline total child anxiety was measured as the sum of child anxiety module CSRs (separation, specific, social, generalized anxiety disorder [GAD]; e.g., Ginsburg, Drake, Tein, Teetsel, \& Riddle, 2015). Prior research using the ADIS-P showed good to excellent test-retest reliability and concurrent validity (Lyneham, Abbott \& Rapee, 2007; Wood, Piacentini, Bergman, McCracken, \& Barrios, 2002). Reliability was assessed on $16 \%$ of all interviews. The intraclass correlation (ICC) for the sum of all anxiety module CSRs was .856.

Parent Anxiety and Depression. The Anxiety Disorders Interview Schedule for DSM-5-Adult \& Lifetime Version (ADIS-5L; Brown \& Barlow, 2014) was utilized to assess parent anxiety disorders and major depressive disorder (MDD). All interviews were completed via telephone. The ADIS-5L assesses for the presence of lifetime and current disorders as well as levels of impairment using a cliniciangenerated CSR. The CSR is rated on a $0-8$ scale, with a rating of 4 indicating clinical impairment. Evaluators were trained on the ADIS-5L through didactics, conducting practice interviews, completing interviews with live supervision, and achieving 
reliability with clinical supervisors. Total PP and CP lifetime anxiety were measured as the sum of the ADIS-5L anxiety module CSRs (panic, GAD, social, separation, specific). The ADIS-5L demonstrates good reliability and inter-rater agreement (Brown, DiNardo, Lehman, \& Campbell, 2001). Reliability was assessed on 20\% of all interviews. For PP interviews, ICCs ranged from .93-.94. For CP interviews, ICCs ranged from $.80-.89$.

Attendance. All PPs and CPs were encouraged to attend all sessions. For the purpose of this study, attendance was measured as the number of hours of treatment attended in person by the PP or CP. Primary parents who missed sessions were encouraged to review the missed session material via telephone with parent group leaders prior to the next group session.

Homework Completion. Parent-reported homework completion was collected from PPs starting in the fifth cohort (out of twelve cohorts). Homework completion was measured via a single item ("How much homework did you complete?") on a 7-point Likert scale $(0=0 \% /$ none of it to $6=100 \% /$ all of $i t)$. We added this homework completion question while data collection was ongoing. As a result these data are available for a subgroup $(n=101)$ of PPs.

Treatment Satisfaction. Following treatment and the post-assessment visit, the same subgroup $(\mathrm{n}=101)$ of PPs that responded to the homework completion measure completed a 7-point Likert-scale satisfaction survey $(1=$ not at all effective/helpful to 7 = extremely effective/helpful). Treatment satisfaction ratings were collected from PP participants starting in the fifth (of twelve) cohorts. Parents answered questions concerning the extent to which they believed their children's 
behavior improved as a function of the treatment, if they would recommend the program, the extent to which the group leaders were helpful, and the extent to which they found treatment components to be beneficial.

We used principal components analysis to explore the factor structure of the satisfaction questionnaire. The extraction revealed three components with eigenvalues above one. However, upon examining the scree plot, the eigenvalues leveled off after the first component. Therefore, a one-component solution, which explained $47.7 \%$ of the variance (eigenvalue $=6.19$ ), was preferred. As such, no rotation was necessary. To avoid introducing unnecessary measurement error, two items with loadings below 0.3 were dropped from the initial 13 items and a new component solution was computed. The subsequent extraction revealed one component (11 items), which explained $56.5 \%$ of the variance (eigenvalue $=6.22$ ). A composite satisfaction score was calculated by averaging the items. Internal consistency for the current study was strong $(\alpha=.92)$.

\section{Data Analytic Plan}

Structural equation modeling was performed using the lavaan package (Rosseel, 2012) version 1.15 in R (R Core Team, 2014). Full information maximum likelihood procedures were used to account for missingness and Huber-White standard errors were used to correct for non-normality and heteroscedasticity (Enders, 2001; Satorra \& Bentler, 2001). Multi-group analysis was used to test whether associations between variables of interest were moderated by treatment group (Turtle/CLK). A separate model was run for each domain of engagement. Path modeling was used to examine predictors of $\mathrm{CP}$ attendance. To refrain from capturing 
the effects of attrition, we left all drop outs' data points as missing. There were no significant differences between analyses that included these data points or left them as missing.

Model fit was assessed using the comparative fit index (CFI), model $x^{2}$ statistics, and the root mean square error of approximation (RMSEA). Acceptable data-model fit is supported by CFI values $>.95$ and RMSEA values $<.05$ (Bentler, 1990; Kline, 2011). For each multi-group model, a model with all paths freed was compared to models with each path constrained, one by one. A scaled chi-square difference test (Satorra \& Bentler, 2001) was completed to compare models with each path constrained one at a time to the fully unconstrained model. Once all paths were tested, we constructed a final model taking into account the chi-square difference test results. If a constrained path was found to significantly worsen model fit, that path was allowed to remain free. Otherwise, it was constrained to be equal across groups in the final model.

Relevant parent and child demographic factors (child/parent age, child/parent ethnicity, child/parent race, parent income, and child/parent sex) were assessed to determine whether they were significantly associated with the dependent variables of interest. These variables were included if a multiple regression containing all potential covariates indicated significant associations with the dependent variable. 
Chapter 3: Results

\section{Preliminary Analyses}

Sample demographic and clinical characteristics can be found in Table 1. Differences in demographic variables between groups were examined in R. Results indicated that there were no differences between the groups on demographic variables. When we examined demographic and clinical variables that significantly predicted engagement outcomes, we found that no clinical or demographic variables predicted PP or CP attendance (all p-values $>.05$ ). Child ethnicity predicted PP homework completion, such that PPs of Hispanic/Latinx children completed relatively less homework compared to other groups $(p=.027)$. Family income predicted PP treatment satisfaction, such that families with an income below $\$ 150,000$ were more satisfied with treatment than families with an greater than or equal to $\$ 150,000(p=.043)$. Primary parent race was associated with missingness in the PP ADIS modules, and was thus included in analyses as a covariate.

\section{Primary Parent Treatment Attendance}

A summary of all PP multigroup analyses can be found in Table 2. Based on $\Delta x^{2}$ tests, we constrained the paths for PP race, PP MDD, PP anxiety, and the interaction between child anxiety and PP anxiety to be equal across groups (all pvalues $>.05) . \Delta x^{2}$ tests revealed that the paths for the intercept and child anxiety should remain freed across groups. Although the $\Delta x^{2}$ test demonstrated a trend in favor of constraining the child anxiety path, we chose to examine the unconstrained model to better understand how child anxiety functioned in each group. Holding all else constant, there was a significant difference in average PP attendance between the 
two groups, $\Delta x^{2}(1)=13.834, p<.001$, with PPs in Turtle attending more than those in CLK. On average, PPs in Turtle and CLK attended 10.309 and 9.024 hours out of 12 hours of treatment, respectively.

The association between child anxiety and PP attendance significantly differed between treatment groups, $\Delta x^{2}(1)=7.947, p=.005$. In Turtle, greater child anxiety was the only significant predictor of greater PP attendance $(b=.959, S E=$ $.296, \beta=.395, p<.001)$. Parent anxiety did not predict PP attendance in Turtle (see Table 2). In CLK, neither child anxiety nor PP anxiety significantly predicted PP attendance (see Table 2). Parent education, PP MDD, and PP race did not predict PP attendance in either group (all p-values $>.05$ ).

\section{Primary Parent-Reported Homework Completion}

Based on $\Delta x^{2}$ tests, we constrained the paths for PP race, PP MDD, PP anxiety, child anxiety, and the interaction between child and PP anxiety to be equal across groups (all p-values $>.05$ ). $\Delta x^{2}$ tests revealed that the intercept and child age paths should remain freed across groups. Holding all else constant, there was a significant difference in average homework completion between the two groups, $\Delta x^{2}$ $(1)=5.545, p=.018$, with PPs receiving CLK reporting completing significantly more HW on average than PPs in Turtle. On the 7-point Likert scale, PPs in CLK rated their homework completion as a 6.271 ( $~ 87 \%$ homework completed $)$ whereas PPs in Turtle rated their completion as 5.880 ( $\sim 81 \%$ completed $)$. Additionally, there was a difference in the association between child age and PP homework completion between the two groups, $\Delta x^{2}(1)=17.466, \mathrm{p}<.001$. In CLK, older child age significantly predicted more homework completion $(b=.099, S E=.023, \beta=.485, \mathrm{p}$ 
$<.001)$, but this was not significant in Turtle $(b=-.031, S E=.027, \beta=-.175, \mathrm{p}=$ .250). For both groups, child anxiety predicted greater PP homework completion $(b=$ $.250, S E=.099, \beta=.200, p=.012)$.

\section{Primary Parent Treatment Satisfaction}

Based on $\Delta x^{2}$ tests, we constrained the paths for the intercept, PP race, PP anxiety, and income to be equal across groups (all p-values $>.05$ ). Although the $\Delta x^{2}$ test demonstrated a trend in favor of constraining the child anxiety path, the model fit was unsatisfactory $\left(x^{2}(6)=7.694, p=.261, \mathrm{CFI}=.869, \mathrm{RMSEA}=.071\right)$. We thus chose to examine the unconstrained model as it demonstrated better fit $\left(x^{2}(5)=3.336\right.$, $p=.648, \mathrm{CFI}=1.00, \mathrm{RMSEA}=0.00) . \Delta x^{2}$ tests revealed that the paths for PP MDD and the interaction between child and PP anxiety should remain freed across groups. Holding all else constant, there was not a significant difference in treatment satisfaction between treatment groups, $\Delta x^{2}(1)=.418, p=.518$. The association between PP MDD and PP satisfaction significantly differed between treatment groups, $\Delta x^{2}(1)=8.865, p=.003$. In Turtle, PP MDD significantly predicted lower satisfaction $(b=-.234, S E=.102, \beta=-.293, p=.023)$. The interaction between child and PP anxiety predicting satisfaction significantly differed between the treatment groups, $\Delta x^{2}(1)=7.973, p=.005$. In CLK only, the interaction between child and PP anxiety significantly predicted satisfaction $(b=-.325, S E=.119, \beta=-.350, \mathrm{p}=.006)$. Specifically, there was a significant positive association between child anxiety and treatment satisfaction among PPs with low anxiety $(-1 \mathrm{SD} ; b=.636, z=3.681, \mathrm{p}<$ $.001)$. There was no association between child anxiety and treatment satisfaction among PPs with high anxiety ( $+1 \mathrm{SD} ; b=-.064, z=-.346, p=.730)$. 


\section{Co-Parent Treatment Attendance}

A summary of CP multigroup analyses can be found in Table 3. Across treatment groups, only greater $\mathrm{CP}$ anxiety predicted greater $\mathrm{CP}$ attendance $(b=.992$, $S E=.495, \beta=.222, p=.045)$. To examine if CP social anxiety was driving this effect, we split the total CP anxiety composite into the social anxiety CSR and the total of all other anxiety CSRs (GAD, panic, separation, and specific). We found that social anxiety was indeed driving this effect, in that greater CP social anxiety significantly predicted greater CP attendance across treatment conditions $(b=1.388$, $S E=.418, \beta=.310, p=.001)$, and the total of all other anxiety module CSRs did not significantly predict $\mathrm{CP}$ attendance $(b=.224, S E=.527, \beta=.050, p=.671)$. None of the interactions between child, $\mathrm{PP}$, and $\mathrm{CP}$ anxiety significantly predicted $\mathrm{CP}$ attendance (all p-values $>.05$ ). 
The current study is among the first to examine parent-, child-, and treatmentlevel factors as independent and interactive predictors of parent engagement in early interventions for BI. We specifically compared Cool Little Kids (a parent-only PT group) and the Turtle Program (which includes simultaneous parent and child groups and in-vivo coaching). We conceptualized primary parent engagement via three different indices: attendance, and parent-reported homework completion and treatment satisfaction. We also extended the literature by exploring the independent and interactive effects of co-parent, primary parent, and child factors in predicting coparent attendance across groups. Findings, implications for clinical application, and recommendations for future directions are discussed below.

In support of our hypotheses and in line with the literature on parent engagement in PT for externalizing problems, primary parents of children with greater lifetime anxiety severity reported completing more homework regardless of treatment format. Indeed, parents of children with more severe anxiety may be more concerned about their children, and thus, be more motivated to practice the skills they learned in treatment sessions at home. Additionally, parents of more anxious children may experience more natural opportunities to practice confronting anxiety-provoking situations between sessions.

Several of our findings highlight the potential benefits of offering multi-modal treatment (i.e., parent-child rather than parent-only) to families with children at-risk for anxiety. For example, although there were high rates of attendance across both groups, primary parents in Turtle exhibited more attendance on average compared to 
those in CLK. Further, greater child anxiety severity predicted more primary parent attendance, but only in Turtle. Research suggests that parents of children with greater anxiety severity expect their children to exhibit more avoidance and anxiety when reacting to anxiety-provoking situations (Wheatcroft \& Creswell, 2007). Thus, parents of more anxious children may have found Turtle's concurrent child group to be especially relevant to their children's distress in social situations. Further, primary parents of highly anxious children may have appreciated the opportunity to practice exposures in session through in-vivo coaching, prompting more attendance at treatment sessions.

However, results regarding treatment satisfaction indicate possible obstacles to engaging a subset of families in multi-modal treatments. Overall, there was not a significant difference in treatment satisfaction between the two groups. In accordance with prior research demonstrating that parents with elevated depressive symptoms report greater dissatisfaction with PT for externalizing (Furey \& Basili, 1988), higher primary parent lifetime depressive symptom severity predicted lower treatment satisfaction, but only in Turtle. Despite more in-vivo support in Turtle, primary parents with a lifetime history of depressive symptoms may have found the active learning and performance aspects of coaching to be distressing. Further, these primary parents may have found it particularly burdensome to complete the special time homework in addition to between-session exposures. The dysfunctional attitudes and attributions that may persist after depression remission (Williams, Healy, Teasdale, White, \& Paykel, 1990) could have also contributed to negative impressions of treatment and subsequent lower satisfaction ratings. Although findings 
suggest that primary parents with a lifetime history of depressive symptoms were less satisfied with Turtle after completing the program, it is important to note that primary parent lifetime depressive symptom severity did not predict either primary parent attendance or homework completion. This indicates that other than being less satisfied with Turtle after completing treatment, parents with a lifetime history of more severe depressive symptoms did not demonstrate any notable difficulties actively engaging in the Turtle Program according to other indices.

Several of our findings underscore potential benefits of a parent-only format for a subset of parents and their inhibited young children. First, results revealed that parents with less lifetime anxiety who have more anxious children were more satisfied with treatment when randomly assigned to CLK. One possibility is that primary parents with low anxiety may have felt that the skills taught in CLK were relevant to their children's needs, and that they were able to implement these skills relatively easily outside of treatment. These primary parents, being low in anxiety, may have felt that a parent-only format was sufficient for them to confidently implement between-session homework and help their children. Second, in line with prior research (Nix et al., 2009), older child age predicted more primary parentreported homework completion, but surprisingly only in CLK. Older children may participate in more extracurricular activities and attend more social gatherings, allowing for more opportunities to practice exposures. However, it is unclear as to why this was only the case in CLK. Third, although results demonstrated that primary parents in CLK reported completing more homework on average than those in Turtle, the difference in the intercept estimates, .391, was less than 1 standard error, 
indicating that this mean difference in average homework completion may have been very small and should be interpreted with caution. Thus, replication of this finding is warranted.

In exploring predictors of co-parent attendance, results indicated that lifetime co-parent social anxiety severity predicted increased co-parent attendance across treatment groups. Such findings stand in contrast to the extant research suggesting that parent psychopathology predicts lower parent engagement in child treatment. However, it is important to note that the majority of the existing research focuses almost exclusively on mothers and, in particular, maternal depression. Evidence has consistently linked parent anxiety with parent threat expectations of child anxious behaviors, suggesting that anxious parents are more likely to perceive themselves as having less control over their anxious children's avoidant and anxious behaviors (Wheatcroft \& Creswell, 2007). Socially anxious co-parents may have less confidence in their children's ability to cope and their ability to support their children, prompting greater treatment attendance. On the other hand, one might expect that socially anxious parents would find it anxiety provoking to verbally and behaviorally engage in treatments with a group format or those that involve in-vivo coaching. Indeed, worries regarding others' judgments are one of the top treatment barriers for adults with social anxiety (Olfson et al., 2000). However, in our study, co-parent social anxiety was associated with increased attendance, perhaps due to increased concern about the child's difficulties. Given that this study did not include an examination of treatment-level predictors of co-parent attendance (as we did for primary parents) due to concerns about power to detect a four-way interaction, future 
research is needed focusing on which treatment formats best engage co-parents, especially those with a history of anxiety.

Interestingly, these results indicate that there may be different processes underlying primary parent and co-parent attendance, as elevated primary parent anxiety severity was not associated with any index of treatment engagement, whereas greater co-parent social anxiety severity predicted more co-parent attendance. Primary parents may attend treatment regardless of their own anxiety given their regular exposure to their children's impairment. On the other hand, co-parents may attend treatment more if they are socially anxious, as they may be more concerned about how to best help their inhibited children or require more guidance in supporting their children. These results highlight the need to consider both parents' engagement in future studies.

\section{Limitations and Future Directions}

The current study had several notable limitations, including the relatively high SES of the sample. Though evaluations of child anxiety treatments have not steadily demonstrated an association between SES and parent engagement, research has consistently indicated that SES is a predictor of parent engagement in PT programs for child externalizing problems (Haine-Schlagel \& Walsh, 2015). Families in this study demonstrated high parent engagement overall across all three indices (attendance, homework completion and satisfaction), which may in part be a function of the high SES of the sample. Future research incorporating samples with a wider income range may help ascertain the influence of SES on parent engagement in PT for child anxiety. Additionally, the high attendance rate may also be due to the 
supports available to families in this efficacy study that are not typically available or feasible in community-based research (e.g., routine reminder emails, provision of free childcare for the target child and siblings). Furthermore, though this study sample was ethnically and racially diverse, it is important to note that there are critical differences between families who participate in efficacy studies and those seen in community settings. Further research disseminating and implementing these interventions in under-resourced settings is crucial in discerning how to best engage families seeking services in the community and whether there is significant added value in implementing a more intensive treatment in community settings (Mian, 2014).

Limitations also include the single question measure of homework completion that was reported by parents following treatment, as parents may have misrepresented or simply estimated the amount of homework they completed. Future research will benefit from careful collection of homework at each session in order to examine homework completion and quality. Moreover, only primary parents who completed treatment provided satisfaction and homework data, possibly resulting in a biased sample. Further, in regard to co-parent analyses, we only analyzed data of co-parents who elected to participate in the study and were married. Additionally, it is impossible to disentangle the effects of the child group and in-vivo coaching in Turtle. Future research should include a child-only treatment comparison to completely isolate and examine the unique effects of specific treatment components. Finally, though there are meaningful differences in the predictors of engagement based on treatment group, there may be other child- and parent-level factors that interact with treatment format to drive parent engagement that were not examined in 
the current study (e.g., parent personality, attributions about their children's anxiety, expectations for treatment, etc.). Future research examining other important childand parent-level factors could lead to important techniques to improve parent engagement. Nevertheless, this study adds to the very limited literature examining two active treatments and is strengthened by the examination of multiple indices of engagement and the inclusion of co-parents (i.e., fathers) in addition to primary parents.

\section{Conclusion}

Considering the central role of parenting in early intervention programs, a deeper understanding of factors associated with parent treatment engagement is crucial to the effectiveness of early interventions developed to mitigate the negative sequelae associated with early childhood BI. This study is one of the first attempts to examine predictors of parent engagement in early interventions for youth at-risk for later anxiety by virtue of $\mathrm{BI}$ and is a starting point for investigating ways to personalize early interventions to enhance engagement. Our findings suggest that the use of a child-group and in-vivo coaching may be powerful tools in engaging parents of highly anxious children. Furthermore, findings underscore the importance of initiating a discussion regarding expectations for treatment when implementing intensive interventions with parents with a history of depressive symptoms. Future work determining which treatment formats are most beneficial in engaging parents will further assist efforts to guide families in choosing an appropriate treatment for their anxious young children. Continued efforts to research predictors of parent engagement are requisite to extend the reach of effective early-intervention programs. 


\section{Appendix}

Table 1. Primary parent, co-parent, and child demographic characteristics.

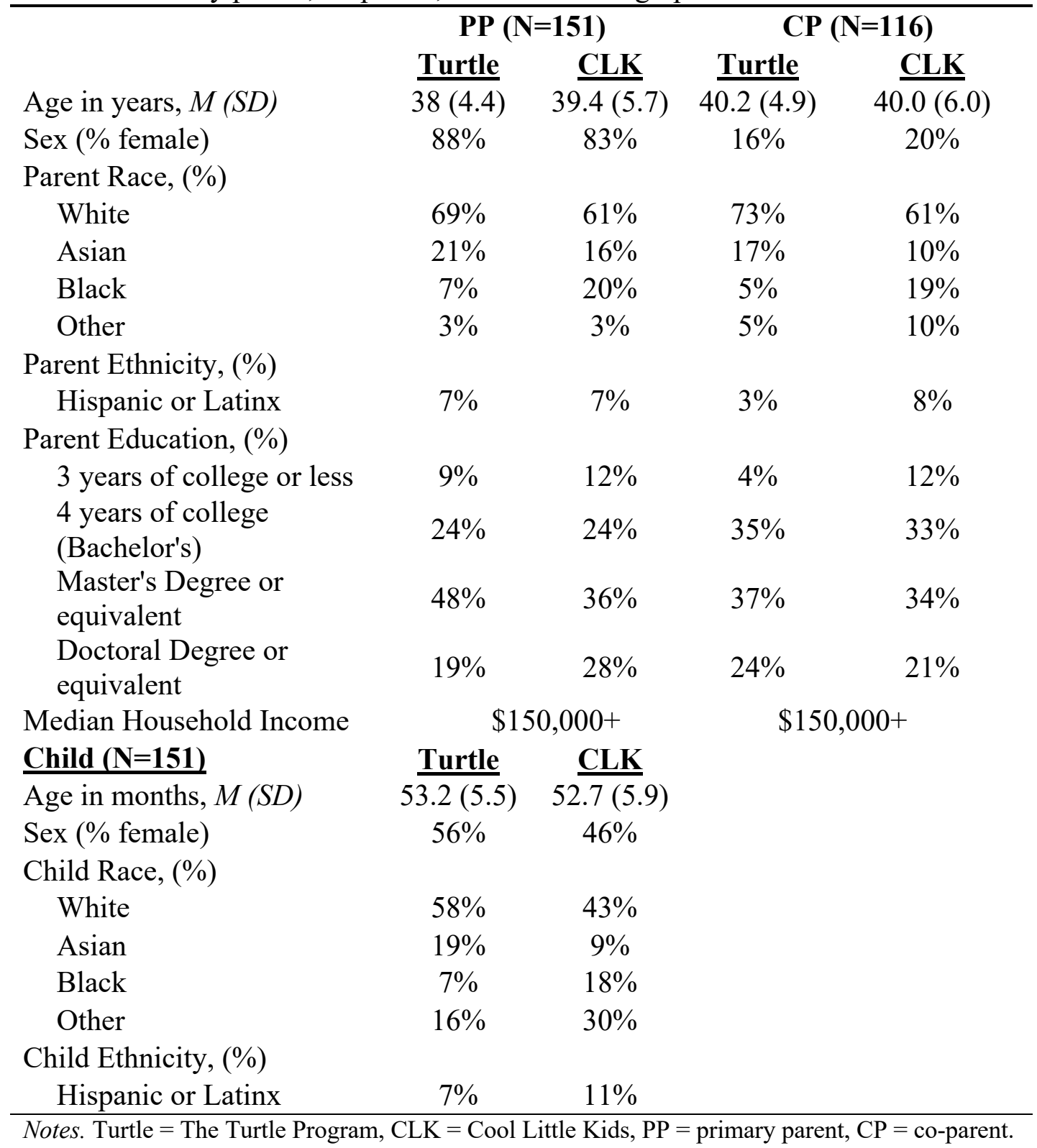


Table 2. Summary of results from primary parent multigroup analyses.

\begin{tabular}{|c|c|c|c|c|c|c|}
\hline & \multicolumn{2}{|c|}{ PP Attendance } & \multicolumn{2}{|c|}{ PP Homework } & \multicolumn{2}{|c|}{ PP Satisfaction } \\
\hline & $b(\mathrm{SE})$ & $\beta$ & $b(\mathrm{SE})$ & $\beta$ & $b(\mathrm{SE})$ & $\beta$ \\
\hline \multicolumn{7}{|l|}{$\underline{\text { Turtle }}$} \\
\hline Child age & & & $-.031(.027)$ & -.175 & & \\
\hline Child Hispanic/Latinx & & & $-.611(.308)^{*}$ & $-.145^{*}$ & & \\
\hline Income & & & & & $.499(.160)^{*}$ & $.366^{*}$ \\
\hline PP African American & $-.271(.519)$ & -.033 & $-.143(.372)$ & -.034 & $-.074(.238)$ & -.026 \\
\hline PP Asian/Other & $.604(.394)$ & .126 & $-.174(.248)$ & -.082 & $-.195(.171)$ & -.136 \\
\hline LT PP MDD & $-.283(.210)$ & -.131 & $-.113(.104)$ & -.095 & $-.234(.102)^{*}$ & $.293 *$ \\
\hline Child total anxiety & $.959(.296)^{*}$ & $.395^{*}$ & $.250(.099)^{*}$ & $.204 *$ & $-.110(.117)$ & -.134 \\
\hline PP total LT anxiety & $.402(.220)$ & .161 & $.027(.116)$ & .021 & $-.105(.100)$ & -.119 \\
\hline Child x PP anxiety & $-.152(.159)$ & -.050 & $-.015(.096)$ & -.008 & $.076(.145)$ & .059 \\
\hline Intercept & $10.309(.244)$ & 5.051 & $5.880(.361)$ & 5.943 & $5.850(.104)$ & 8.815 \\
\hline \multicolumn{7}{|l|}{$\underline{\text { CLK }}$} \\
\hline Child age & & & $.099(.023)^{* *}$ & $.485^{* *}$ & & \\
\hline Child ethnicity & & & $-.611(.308)^{*}$ & $-.188^{*}$ & & \\
\hline Income & & & & & $.499(.160)^{*}$ & $.275^{*}$ \\
\hline PP African American & $-.271(.519)$ & -.051 & $-.143(.372)$ & -.049 & $-.074(.238)$ & -.032 \\
\hline PP Asian/Other & $.604(.394)$ & .114 & $-.174(.248)$ & -.066 & $-.195(.171)$ & -.094 \\
\hline PP MDD & $-.283(.210)$ & -.140 & $-.113(.104)$ & -.105 & $.047(.112)$ & .055 \\
\hline Child total anxiety & $.008(.204)$ & .004 & $.250(.099)^{*}$ & $.200^{*}$ & $.281(.152)$ & .286 \\
\hline PP total anxiety & $.402(.220)$ & .219 & $.027(.116)$ & .025 & $-.105(.100)$ & -.126 \\
\hline Child x PP anxiety & $-.152(.159)$ & -.113 & $-.015(.096)$ & -.013 & $-.325(.119)^{*}$ & $.350^{*}$ \\
\hline Intercept & $9.024(.307)$ & 4.293 & $6.271(.427)$ & 5.558 & $5.850(.104)$ & 6.601 \\
\hline \multicolumn{7}{|l|}{ Model fit } \\
\hline$x^{2}$ & \multicolumn{2}{|c|}{$x^{2}(5)=2.587, p=.763$} & \multicolumn{2}{|c|}{$x^{2}(8)=8.127, p=.421$} & \multicolumn{2}{|c|}{$x^{2}(5)=3.336, p=.648$} \\
\hline CFI & \multicolumn{2}{|c|}{1.00} & \multicolumn{2}{|c|}{0.99} & \multicolumn{2}{|c|}{1.00} \\
\hline RMSEA & \multicolumn{2}{|c|}{0.00} & \multicolumn{2}{|c|}{.016} & \multicolumn{2}{|c|}{0.00} \\
\hline
\end{tabular}

Notes. Turtle $=$ The Turtle Program, $\mathrm{CLK}=$ Cool Little Kids,, $\mathrm{PP}=$ primary parent, $\mathrm{LT}=$ lifetime, $\mathrm{MDD}=$ Major Depressive Disorder, $\mathrm{CP}=$ co-parent, ${ }^{* *}=p<.001,{ }^{*}=p<.05$. 
Table 3. Summary of results from co-parent multigroup analyses

\begin{tabular}{llc}
\hline & \multicolumn{2}{c}{ CP Attendance } \\
\hline & \multicolumn{1}{c}{$b(\mathrm{SE})$} & $\beta$ \\
\hline PP African American & $-1.755(.914)$ & -.120 \\
PP Asian/Other & $-.405(.977)$ & -.039 \\
CP MDD & $-.291(.440)$ & -.065 \\
PP MDD & $-.337(.444)$ & -.076 \\
Child total anxiety & $-.079(.434)$ & -.018 \\
CP social anxiety & $1.275(.465)^{*}$ & $.285^{*}$ \\
PP total anxiety & $.455(.627)$ & .101 \\
Child x PP anxiety x & $-.094(.327)$ & -.048 \\
CP social anxiety & $5.740(.513)$ & 1.291 \\
Intercept & Notes. PP $=$ primary parent, CP $=$ co-parent, MDD $=$ Major Depressive \\
Disorder, ${ }^{* *}=p<.001,{ }^{*}=p<.05^{*}$. &
\end{tabular}




\section{References}

Arendt, K., Thastum, M., \& Hougaard, E. (2016). Homework adherence and cognitive behaviour treatment outcome for children and adolescents with anxiety disorders. Behavioural and Cognitive Psychotherapy, 44(2), 225235.

Armbruster, P., \& Kazdin, A. E. (1994). Attrition in child psychotherapy. Advances in Clinical Child Psychology, 16, 81-108.

Bagner, D. M., \& Eyberg, S. M. (2003). Father Involvement in Parent Training: When Does It Matter? Journal of Clinical Child and Adolescent Psychology, 32(4), 599-605.

Bentler, P. M. (1990). Comparative fit indexes in structural models. Psychological Bulletin, 107(2), 238-246.

Bishop, G., Spence, S. H., \& McDonald, C. (2003). Can parents and teachers provide a reliable and valid report of behavioral inhibition?. Child Development, 74(6), 1899-1917.

Brown, T. A., \& Barlow, D. H. (2014). Anxiety and Related Disorders Interview Schedule for DSM-5 (ADIS-5L): Lifetime Version. Client Interview Schedule. Oxford University Press.

Brown, T. A., Di Nardo, P. A., Lehman, C. L., \& Campbell, L. A. (2001). Reliability of DSM-IV anxiety and mood disorders: implications for the classification of emotional disorders. Journal of Abnormal Psychology, 110(1), 49. 
Calvert, S. C., \& Johnston, C. (1990). Acceptability of treatments for child behavior problems: Issues and implications for future research. Journal of Clinical Child Psychology, 19(1), 61-74.

Carpenter, A. L., Puliafico, A. C., Kurtz, S. M., Pincus, D. B., \& Comer, J. S. (2014). Extending parent-child interaction therapy for early childhood internalizing problems: New advances for an overlooked population. Clinical Child and Family Psychology Review, 17(4), 340-356.

Cartwright-Hatton, S., McNally, D., Field, A. P., Rust, S., Laskey, B., Dixon, C., ... \& Symes, W. (2011). A new parenting-based group intervention for young anxious children: Results of a randomized controlled trial. Journal of the American Academy of Child \& Adolescent Psychiatry, 50(3), 242-251.

Chronis-Tuscano, A., Degnan, K. A., Pine, D. S., Perez-Edgar, K., Henderson, H. A., Diaz, Y., ... Fox, N. A. (2009). Stable early maternal report of behavioral inhibition predicts lifetime social anxiety disorder in adolescence. Journal of the American Academy of Child \& Adolescent Psychiatry, 48(9), 928-935.

Chronis-Tuscano, A., Danko, C. M., Rubin, K. H., Coplan, R. J., \& Novick, D. R. (2018). Future directions for research on early intervention for young children at risk for social anxiety. Journal of Clinical Child and Adolescent Psychology, 47(4), 655-667.

Chronis-Tuscano, A., Rubin, K. H., O’Brien, K. A., Coplan, R. J., Thomas, S. R., Dougherty, L. R., ... \& Menzer, M. (2015). Preliminary evaluation of a multimodal early intervention program for behaviorally inhibited preschoolers. Journal of Consulting and Clinical Psychology, 83(3), 534. 
Connell, A. M., \& Goodman, S. H. (2002). The association between psychopathology in fathers versus mothers and children's internalizing and externalizing behavior problems: A meta- analysis. Psychological Bulletin, 128, 746-773.

Coplan, R. J., Schneider, B. H., Matheson, A., \& Graham, A. (2010). 'Play skills' for shy children: development of a Social Skills Facilitated Play early intervention program for extremely inhibited preschoolers. Infant and Child Development, 19(3), 223-237.

Danko, C. M., O’Brien, K. A., Rubin, K. H., \& Chronis-Tuscano, A. (2019). The Turtle Program: PCIT for young children displaying behavioral inhibition. In L. N. Niec (Ed.), Handbook of parent-child interaction therapy: Innovations and applications for research and practice. New York, NY: Springer.

Degnan, K. A., \& Fox, N. A. (2007). Behavioral inhibition and anxiety disorders: Multiple levels of a resilience process. Development and Psychopathology, 19, 729-746.

Eaves, L. C., Wingert, H. D., Ho, H. H., \& Mickelson, E. C. (2006). Screening for autism spectrum disorders with the social communication questionnaire. Journal of Developmental \& Behavioral Pediatrics, 27(2), S95S103.

Enders, C. K. (2001). The impact of nonnormality on full information maximumlikelihood estimation for structural equation models with missing data. Psychological Methods, 6(4), 352-370.

Eyberg, S. (1988). Parent-child interaction therapy: Integration of traditional and behavioral concerns. Child \& Family Behavior Therapy, 10(1), 33-46. 
Fabiano, G. A., Chacko, A., Pelham, W. E., Jr., Robb, J., Walker, K. S., Wymbs, F., Pirvics, L. (2009). A comparison of behavioral parent training programs for fathers of children with attention-deficit/hyperactivity disorder. Behavior Therapy, 40(2), 190-204.

Furey, W. M., \& Basili, L. A. (1988). Predicting consumer satisfaction in parent training for noncompliant children. Behavior Therapy, 19(4), 555-564.

Ginsburg, G. S., Drake, K. L., Tein, J., Teetsel, R., \& Riddle, M. A. (2015). Preventing onset of anxiety disorders in offspring of anxious parents: A randomized controlled trial of a family-based intervention. The American Journal Of Psychiatry, 172(12), 1207-1214.

Gonzalez, A., Weersing, V. R., Warnick, E. M., Scahill, L. D., \& Woolston, J. L. (2011). Predictors of treatment attrition among an outpatient clinic sample of youths with clinically significant anxiety. Administration and Policy in Mental Health and Mental Health Services Research, 38(5), 356-367.

Haine-Schlagel, R., \& Walsh, N. E. (2015). A review of parent participation engagement in child and family mental health treatment. Clinical Child and Family Psychology Review, 18(2), 133-150.

Kagan, J., Reznick, J. S., Snidman, N., Gibbons, J., \& Johnson, M. O. (1988). Childhood derivatives of inhibition and lack of inhibition to the unfamiliar. Child Development, 59(6), 1580-1589.

Kazantzis, N., Whittington, C., \& Dattilio, F. (2010). Meta-analysis of homework 
effects in cognitive and behavioral therapy: A replication and extension. Clinical Psychology: Science and Practice, 17, 144-156.

Kazdin, A. E. (2000). Perceived barriers to treatment participation and treatment acceptability among antisocial children and their families. Journal of Child and Family Studies, 9(2), 157-174.

Kendall, P. C., \& Sugarman, A. (1997). Attrition in the treatment of childhood anxiety disorders. Journal of Consulting and Clinical Psychology, 65(5), 883888.

Kline, R. B. (2011). Convergence of structural equation modeling and multilevel modeling. In M. Williams \& W. Vogt, The SAGE Handbook of Innovation in Social Research Methods (pp. 562-589).

Last, C. G., Hersen, M., Kazdin, A. E., Orvaschel, H., \& Perrin, S. (1991). Anxiety disorders in children and their families. Archives of General Psychiatry, 48(10), 928-934.

Loren, R. E. A., Ciesielski, H. A., \& Tamm, L. (2017). Behavioral parent training groups for ADHD in clinical settings: Does offering a concurrent child group add value? Clinical Practice in Pediatric Psychology, 5(3), 221-231.

Lyneham, H. J., Abbott, M. J., \& Rapee, R. M. (2007). Interrater reliability of the Anxiety Disorders Interview Schedule for DSM-IV: child and parent version. Journal of the American Academy of Child \& Adolescent Psychiatry, 46(6), 731-736.

Mian, N. D. (2014). Little children with big worries: Addressing the needs of young, 
anxious children and the problem of parent engagement. Clinical Child and Family Psychology Review, 17(1), 85-96.

Miller, D. L., \& Kelley, M. L. (1992). Treatment acceptability: The effects of parent gender, marital adjustment, and child behavior. Child \& Family Behavior Therapy, 14(1), 11-23.

Monga, S., Rosenbloom, B. N., Tanha, A., Owens, M., \& Young, A. (2015).

Comparison of child-parent and parent-only cognitive-behavioral therapy programs for anxious children aged 5 to 7 years: Short-and long-term outcomes. Journal of the American Academy of Child \& Adolescent Psychiatry, 54(2), 138-146.

Nix, R. L., Bierman, K. L., \& McMahon, R. J. (2009). How attendance and quality of participation affect treatment response to parent management training. Journal of Consulting and Clinical Psychology, 77(3), 429-438.

Olfson, M., Guardino, M., Struening, E., Schneier, F. R., Hellman, F., \& Klein, D. F. (2000). Barriers to the treatment of social anxiety. The American Journal of Psychiatry, 157(4), 521-527.

Pereira, A. I., Muris, P., Mendonça, D., Barros, L., Goes, A. R., \& Marques, T. (2016). Parental involvement in cognitive-behavioral intervention for anxious children: Parents' in-session and out-session activities and their relationship with treatment outcome. Child Psychiatry and Human Development, 47(1), $113-123$.

Podell, J. L., \& Kendall, P. C. (2011). Mothers and fathers in family cognitive- 
behavioral therapy for anxious youth. Journal Of Child And Family

Studies, 20(2), 182-195.

Prinz, R. J., \& Miller, G. E. (1994). Family-based treatment for childhood antisocial behavior: Experimental influences on dropout and engagement. Journal of Consulting and Clinical Psychology, 62(3), 645-650.

R Core Team (2014). R: A language and environment for statistical computing. $R$ Foundation for Statistical Computing, Vienna, Austria. URL http://www.Rproject.org/

Rapee, R. M., Kennedy, S., Ingram, M., Edwards, S., \& Sweeney, L. (2005). Prevention and early intervention of anxiety disorders in inhibited preschool children. Journal of Consulting and Clinical Psychology, 73(3), 488.

Reid, M. J., Webster-Stratton, C., \& Baydar, N. (2004). Halting the development of conduct problems in Head Start children: The effects of parent training. Journal of Clinical Child and Adolescent Psychology, 33, 279-291.

Rosseel, Yves (2012). lavaan: An R Package for Structural Equation Modeling. Journal of Statistical Software, 48(2), 1-36. URL http://www.jstatsoft.org/v48/i02/.

Rubin, K. H., Coplan, R. J., \& Bowker, J. C. (2009). Social withdrawal in childhood. Annual Review of Clinical Psychology, 60, 141-171.

Satorra, A., \& Bentler, P. M. (2010). Ensuring positiveness of the scaled difference chi-square test statistic. Psychometrika, 75(2), 243-248. 
Serketich, W., \& Dumas, J. (1996). The effectiveness of behavioral parent training to modify antisocial behavior in children: A meta-analysis. Behavior Therapy, $27,171-186$.

Staudt, M. (2007). Treatment engagement with caregivers of at-risk children: Gaps in research and conceptualization. Journal of Child and Family Studies, 16, 183196.

Tynan, W. D., Schuman, W., \& Lampert, N. (1999). Concurrent parent and child therapy groups for externalizing disorders: From the laboratory to the world of managed care. Cognitive and Behavioral Practice, 6, 3-9.

Waters, A. M., Ford, L. A., Wharton, T. A., \& Cobham, V. E. (2009). Cognitivebehavioural therapy for young children with anxiety disorders: Comparison of a child + parent condition versus a parent only condition. Behaviour Research and Therapy, 47(8), 654-662.

Wheatcroft \& Creswell (2007). Parents' cognitions and expectations about their preschool children: The contribution of parental anxiety and child anxiety. British Journal of Developmental Psychology, 25, 435-441.

Williams, J. M., Healy, D., Teasdale, J. D., White, W., \& Paykel, E. S. (1990). Dysfunctional attitudes and vulnerability to persistent depression. Psychological Medicine, 20(2), 375-381.

Wood, J. J., Piacentini, J. C., Bergman, R. L., McCracken, J., \& Barrios, V. (2002). Concurrent validity of the anxiety disorders section of the anxiety disorders interview schedule for DSM-IV: child and parent versions. Journal of Clinical Child and Adolescent Psychology, 31(3), 335-342. 